\title{
Effect of number and relatedness of stimulus-term components on paired-associate learning'
}

\author{
JOSEPH LIFTIK ${ }^{2}$ and KENNETH L. LEICHT, Ilinois State \\ University, Normal, Ill. 61761
}

Word compounds comprised of two or three conceptually related or two or three unrelated words formed the stimulus terms in four paired-associate (PA) lists. List learning was more rapid when stimuli were compounds of conceptually related words than when stimuli consisted of unrelated words. An effect of number of words in the compounds and the interaction of number and relatedness of compound words were not obtained. Results were interpreted in terms of stimulus selection.

Use of only a portion of the presented PA stimulus as a recall cue is now an established phenomenon (Cohen \& Musgrave, 1964). Stimulus selection appears to occur whenever stimuli are poorly integrated units, e.g., GYX, which cannot easily be responded to as a whole (Leicht \& Kausler, 1965). Selection circumvents learning the entire stimulus compound. Nevertheless, time spent searching the compound for a suitable recall cue should slow overall learning rate. The assumption that increasing the number of components in the stimulus slows learning by increasing selection time was examined by comparing learning of PA lists containing stimuli comprised of either two or three words. In addition, component words were either conceptually related, e.g., OAK, ELM, or were unrelated, e.g., OAK, BOY. Stimuli whose components are conceptually related tend to elicit a single category-name associate which may mediate as a recall cue, the selection process thereby bypassed. Hence, slower learning for three- than for two-word stimuli was expected only when component words were unrelated.

The extent to which differential stimulus selection accounted for an effect of type of stimulus compound on rate of learning was directly assessed through a test of stimulus selection which requires that $S$ produce the response term to components of the stimulus presented individually. In a selection test, $\mathbf{S}$ is presumably able to produce the response term only to component(s) which had served as recall cues in acquisition (Underwood, Ham, \& Ekstrand, 1962).

\section{METHOD}

List stimuli were either two conceptually related words (List 2C), three conceptually related words (List 3C), two unrelated words (List $2 \mathrm{~N}$ ), or three unrelated words (List $3 \mathrm{~N}$ ). Stimulus components were said to be conceptually related if they elicited the same category-name associate in a word-association task (Cohen, Bousfield, \& Whitmarsh, 1957). The same set of single-word responses was used in each list. Each of the four 14-pair lists was learned by a different group of 15 undergraduates.

Lists were presented by the alternate-study-and-recall method until $\mathrm{S}$ achieved one perfect recall. To minimize serial learning, pair order was varied from trial to trial. A $2-\mathrm{sec}$ rate applied for both study and recall portions of trials. The test of stimulus selection was given immediately upon S's achieving criterion and consisted of presenting components of the stimuli individually for $2 \mathrm{sec}$ under instructions to give the response that had gone with the entire compound. For both acquisition and selection phases, materials were presented by a Carousal projector with attached interval timer.

\section{RESULTS AND DISCUSSION}

Mean trials to criterion (SDs in parentheses) was 5.27 (1.84), $6.73(2.21), 4.73(1.53)$, and $5.00(2.19)$ for $2 \mathrm{~N}, 3 \mathrm{~N}$,
2C, and $3 \mathrm{C}$ conditions, respectively. The effect of relatedness of components was significant, $F(1,56)=4.68, p<.05$, suggesting that selection was minimized in $C$ conditions through use of the category name as a recall cue. The effect of number of component words was not reliable, $F(1,56)=2.73$, $p>.05$. Contrary to prediction, there was no evidence that three-word stimuli resulted in slower learning than two-word stimuli only when components were unrelated, $F(1,56)$ for interaction $=1.31, \mathrm{p}<.05$. The same pattern of results emerged when recall from Trials 1 and 2 only was examined. However, a Trials by Number of Components interaction was obtained, $F(1,56)=12.91, p<.001$, such that on Trial 1 number correct was less for two-word than for three-word stimuli while the converse occurred on Trial 2. The interaction suggests that in two-word conditions $\mathrm{S}$ attempted to learn the entire compound on Trial 1 but abandoned the learning strategy for a selection strategy on subsequent trials, whereas in three-word conditions $\mathbf{S}$ may have immediately initiated the selection strategy. Thus, time spent in attempting to learn the entire compound in two-word conditions may have made up for the additional selection time in three-word conditions. The interpretation accounts for the failure to obtain the Number by Relatedness of Components interaction in the trials-tocriterion analysis and is supported by the selection data below.

Analysis of selection data showed more errors (omissions and misplaced responses) in recalling response terms to individual components in $N$ than in $C$ conditions, $F(1,56)=$ $174.08, p<.001$, suggesting again that the category name elicited by $\mathrm{C}$ components served as the recall cue. Although there were more errors in recalling to components of three-word stimuli than to components of two-word stimuli, $F(1,56)=94.01, p<.001$, the effect was apparently an artifact of differential error opportunity (42 possible errors in three-word conditions, 28 in two-word conditions). Total errors was expressed as a proportion of total possible errors for two- and three-word conditions. A $t$ for the difference between the proportions was $1.16, p>.05$, indicating that the significant $F$ for number of components was an artifact. Of particular interest was the Number by Relatedness of Components interaction, $F(1,56)=56.11, \quad p<.001$. As indicated in Fig. 1, the difference between $\mathrm{C}$ and $\mathrm{N}$ conditions

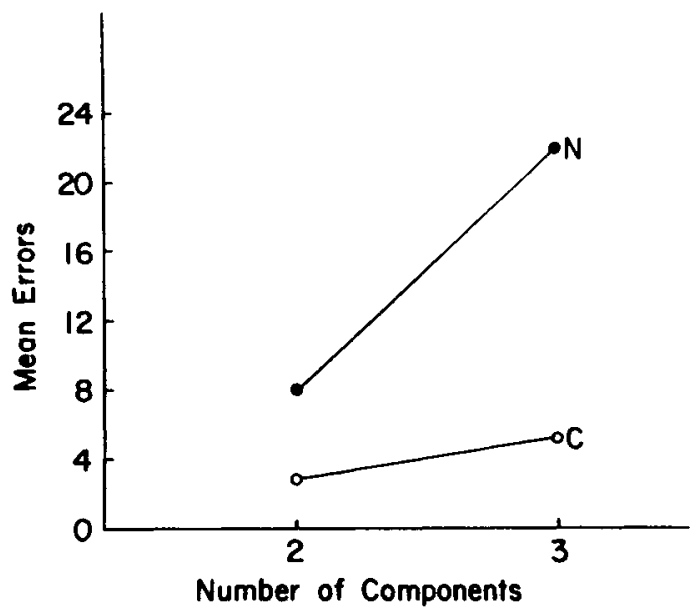

Fig. 1. Mean errors in recalling to separate stimulus components as a function of rehatedness and number of components. 
is larger for three-word than for two-word stimuli. The smaller difference for two-word stimuli would have been obtained had $S$ attempted to learn the entire compound in two-word conditions and supports the interpretation given the failure to obtain the Number by Relatedness of Components interaction in the trials-to-criterion analysis.

\section{REFERENCES}

COHEN, B. H., Bousfield, W. A., \& WhitMaRSh, C. A. Cultural norms for verbal items in 43 categories. Technical Reports No. 22 ONR Contract Nonr-631. University of Conn., Storrs, Conn., 1957.
COHEN, J. D., \& MUSGRAVE, B. S. Effect of meaningfulness on cue selection in paired-associate learning. Journal of Experimental Psychology, 1964, 68, 289-291.

LEICHT, K. L., \& KAUSLER, D. H. Functional stimulus learning as related to degree of practice and meaningfulness. Journal of Experimental Psychology, 1965, 69, 100-101.

UNDERWOOD, B. J., HAM, M., \& EKSTRAND, B. Cue selection in paired-associate learning. Journal of Experimental Psychology, 1962, $64,405-409$.

\section{NOTES}

1. This report was based upon the senior author's Master's thesis.

2. Now at Southern Illinois University, Carbondale. 